Japan. J. Math.

Vol. 28, No. 1, 2002

\title{
A note on regularity of weak solutions of the Navier-Stokes equations in $\mathbf{R}^{n}$
}

\author{
By Luigi C. Berselli
}

(Received April 15, 2001)

(from Tôhoku Mathematical Journal)

\begin{abstract}
In this paper we consider the $n$ dimensional Navier-Stokes equations and we prove a new regularity criterion for weak solutions. More precisely, if $n=$ 3,4 we show that the "smallness" of at least $n-1$ components of the velocity in $L^{\infty}\left(0, T ; L_{w}^{n}\left(\mathbf{R}^{n}\right)\right)$ is sufficient to ensure regularity of the weak solutions.
\end{abstract}

\section{Introduction}

The aim of this paper is to improve a criterion for the regularity of weak solutions of the Navier-Stokes equations in $\mathbf{R}^{n}$ and to give a simpler proof of some known results. We shall consider the system of the Navier-Stokes equations below

$$
\begin{cases}\frac{\partial u}{\partial t}+(u \cdot \nabla) u-\nu \Delta u+\nabla p=f & \text { in } \mathbf{R}^{n} \times(0, T) \\ \nabla \cdot u=0 & \text { in } \mathbf{R}^{n} \times(0, T) \\ u(x, 0)=u_{0}(x) & \text { in } \mathbf{R}^{n},\end{cases}
$$

and to avoid inessential calculation we assume that the external force $f$ vanishes, even if it is easy to include nonzero smooth forces. Here $L^{p}:=\left(L^{p}\left(\mathbf{R}^{n}\right)\right)^{n}$, for $1 \leq p \leq \infty$ with norm $\|\cdot\|_{p}$, is the usual Lebesgue space. It is well-known that if a weak solution belongs to

$$
L^{p}\left(0, T ; L^{q}\right) \text { with } \frac{2}{p}+\frac{n}{q} \leq 1, \text { for } q>n,
$$

then it is regular, see Prodi [18] and Serrin [19] if $2 / p+n / q<1$. For the case with $2 / p+n / q=1$ see for instance Sohr [20]. A recent monograph on the existence and regularity theory for the Navier-Stokes equations, that collects almost all the results cited and used in this paper, is the book of Galdi [7]. The classical result

2000 Mathematics Subject Classification: $35 \mathrm{~B} 65$, 35K55, 76D05.

Key words. Navier-Stokes equations, weak solutions, regularity. 
above, involving the condition (2) (which is present just in the 1934 Leray's paper [13]), does not cover the limit case $p=\infty$ and $q=n$, but the remarkable result of non-existence of Leray's self similar solutions in $L^{3}$ proved in Nečas, Rủžička, and Šverák [17], may also suggest that $L^{\infty}\left(0, T ; L^{3}\right)$ is a regularity class. Recall also that the study of $L^{3}$ solutions is interesting by itself, since it is a starting point in the dimensionfree estimates of Caffarelli, Kohn, and Nirenberg [6].

In this direction there are some partial results, in particular it is known that

$$
L^{\infty}\left(0, T ; L^{n}\right)
$$

is a uniqueness class, see Kozono and Sohr [11]. See also P.-L. Lions and Masmoudi [15]. A little bit stronger condition on the time variable, for instance continuity (Sohr and von Whal [21]), or left continuity (Masuda [16]), or bounded variation (Kozono and Sohr [12]) ensures the regularity. For a review on these results see Kozono [10].

Another condition, ensuring the regularity, is the one involving the so called "Hypothesis A", introduced by Beirão da Veiga [3]. In reference [3] it is proved (for the problem with $n=3,4)$ that if $u \in L^{\infty}\left(0, T ; L^{n}\right)$ satisfies the "Hypothesis A," then $u$ is a strong solution of $(1)$ in $(0, T)$. Observe that, as quite particular cases, Hypothesis A is satisfied if the solution is left-continuous or of bounded variation with values in $L^{n}$ or even if the jumps of the $L^{n}$-norm are small enough. In Beirão da Veiga [4] this result is improved by showing that, if at least $n-1$ components of $u$ satisfy Hypothesis A, then the solution is regular.

On the other hand it is well-known that if the norm of $u$ in $L^{\infty}\left(0, T ; L^{n}\right)$ is small enough, then the solution is smooth. For an elementary proof see [3]. By requiring some smallness it is possible to weaken the condition in the space variables. In Kozono [10] it is also shown that the smallness in

$$
L^{\infty}\left(0, T ; L_{w}^{n}\right)
$$

is enough to have regularity, where $L_{w}^{n}$ is the weak- $L^{n}$ space.

In this paper we give a simple proof of this result and we improve it by showing that only $n-1$ components of the velocity satisfying the latter condition is sufficient to have smoothness of weak solutions.

Our calculations are true at a formal level for any $n$, but the result will follow for $n \leq 4$. In particular, our result encompasses the case $n=3$, which is more interesting from the physical point of view. For $n \geq 5$ it is necessary to resort to more technical methods, see for example Struwe [22]. Recall also (see $\S 4$ in Kato [9]) that $n=4$ is a limit case, just for the problem of existence of weak solutions.

We recall that the divergence-free vector $u$ is a weak (or Leray-Hopf) solution of the Navier-Stokes equations if it has the following regularity properties

$$
u \in C_{w}\left(0, T ; L^{2}\right) \cap L^{2}\left(0, T ; H^{1}\right), \quad \text { with } \frac{\partial u}{\partial t} \in L_{l o c}^{1}\left(0, T ; H^{-1}\right)
$$


if it satisfies

(6)

$$
\int_{0}^{T} \int_{\mathbf{R}^{n}}\left[u \frac{\partial \phi}{\partial t}-\nu \nabla u \cdot \nabla \phi-(u \cdot \nabla) u \phi\right] d x d t=\int_{\mathbf{R}^{n}}\left[u(T) \phi(T)-u_{0} \phi(0)\right] d x
$$

for all divergence-free $\phi \in C^{1}\left(0, T ; H^{1} \cap L^{n}\right)$ and furthermore if the so called "energy inequality" holds:

$$
\frac{1}{2}\|u(t)\|_{2}+\int_{0}^{t}\|\nabla u(s)\|_{2} d s \leq \frac{1}{2}\left\|u_{0}\right\|_{2}, \quad \text { for any } t \geq 0 .
$$

Here $W^{k, p}:=\left(W^{k, p}\left(\mathbf{R}^{n}\right)\right)^{n}$ and $H^{s}:=W^{s, 2}$ are the customary Sobolev spaces. Recall that $C_{w}\left(0, T ; L^{2}\right)$ is the space of weakly continuous functions on $(0, T)$ with values in $L^{2}$.

We say that a weak solution $u$ is strong if it also satisfies:

$$
u \in L^{2}\left(0, T ; H^{2}\right) \cap L^{\infty}\left(0, T ; H^{1}\right), \quad \text { with } \frac{\partial u}{\partial t} \in L^{2}\left(0, T ; L^{2}\right) .
$$

In particular it is well-known that strong solutions are unique in the class of weak solutions. Furthermore, a strong solution is also regular (say a classical solution). Note that, by a standard result of functional analysis, condition (8) implies that $u \in C\left(0, T ; H^{1}\right)$ and, if $n \leq 4$, we have that $H^{1} \subset L^{n}$. Then strong solutions belong to $C\left(0, T ; L^{n}\right)$, that is a regularity class.

We denote by $[.]_{n}$, the quasi-norm of or weak- $L^{n}$ space, see Section 2 for the precise definition of this space and some of its properties. In the sequel we shall need the following definition.

Definition 1. Given a vector $b \in \mathbf{R}^{n}$, we define $\widehat{b}$ as the projection of $b$ onto the hyperplane generated by the first $n-1$ vectors of an orthonormal basis of $\mathbf{R}^{n}$.

The main result we shall prove is the following.

THEOREM 1.1. Let $u_{0} \in H^{1}$, with $\nabla \cdot u_{0}=0$ and let $u$ be a weak solution of the Navier-Stokes equations (1) in $\mathbf{R}^{n} \times(0, T)$, for $n \leq 4$. There exists a positive constant $C_{0}$ such that if

$$
\sup _{0<t<T}[\widehat{u}(t)]_{n}<C_{0}
$$

then the solution $u$ is strong in $(0, T)$.

REMARK 1.2. The positive constant $C_{0}$ depends only on the space dimension $n$ and on the viscosity coefficient $\nu$. Moreover, $C_{0}$ is independent of the initial datum and of the time $T$, see the inequality (13) below. 


\section{Some results regarding Lorentz spaces}

In the sequel we shall use some results related to Lorentz spaces. We briefly recall the definition of such spaces. Given a measurable function $f: \mathbf{R}^{n} \rightarrow \mathbf{R}$ we say that $f \in L^{p, q}$, for $1 \leq p \leq \infty$ and $1 \leq q<\infty$, if

$$
\|f\|_{p, q}:=\left[\int_{0}^{\infty}\left[\sigma\left(\mu\left\{x \in \mathbf{R}^{n}:|f(x)|>\sigma\right\}\right)^{1 / p}\right]^{q} \frac{d \sigma}{\sigma}\right]^{1 / q}<\infty
$$

where $\mu\{$.$\} denotes the Lebesgue measure. With this definition the spaces L^{p, q}$ are not Banach spaces, since $\|\cdot\|_{p, q}$ is just a quasi-norm. If $p>1$ it is possible to define $L^{p, q}$ in an equivalent way so as to make it a Banach space, see Ch. 1 in Bergh and Löfström [5]. We observe that $L^{p, \infty}=L_{w}^{p}$ the Marcinkiewicz (or weak) $L^{p}$ space of measurable functions such that

$$
\|f\|_{L_{w}^{p}}:=[f]_{p}:=\sup _{\sigma>0} \sigma\left(\mu\left\{x \in \mathbf{R}^{n}:|f(x)|>\sigma\right\}\right)^{1 / p}<\infty .
$$

We also recall that, if $r_{1} \leq r_{2}$, then $L^{p, r_{1}} \subset L^{p, r_{2}}$; furthermore $L^{p, p}$ is isomorphic to the usual Lebesgue space $L^{p}$, for $1 \leq p<\infty$.

For our purposes we need the following lemma, which is proved in Kozono [10], Section 2. For the reader's convenience we sketch its proof.

Lemma 2.1. Let $v$ be in $\mathcal{D}^{1,2}:=\left\{v \in L_{l o c}^{1}: \nabla v \in L^{2}\right\}, w$ be in $L^{2}$ and $z$ be in $L_{w}^{n}$. Then

$$
\left|\int_{\mathbf{R}^{n}} v w z d x\right| \leq C_{1}(n)\|\nabla v\|_{2}\|w\|_{2}[z]_{n}
$$

with the constant $C_{1}(n)$ depending only on the space dimension $n$.

Proof. We recall that if $f \in L^{p_{1}, q_{1}}$ and $g \in L^{p_{2}, q_{2}}$ (for $1<p_{1}, p_{2}<\infty$ ) with $1 / p:=1 / p_{1}+1 / p_{2}<1$, then $f g \in L^{p, q}$ with

$$
\|f g\|_{p, q} \leq C\|f\|_{p_{1}, q_{1}}\|g\|_{p_{2}, q_{2}} \text { for } q:=\min \left\{q_{1}, q_{2}\right\} .
$$

By using the above result (a generalization of the classical Hölder inequality) we obtain

$$
\left|\int_{\mathbf{R}^{n}} v w z d x\right| \leq C\|v\|_{\frac{2 n}{n-2}, 2}\|w\|_{2,2}\|z\|_{n, \infty}
$$

We now observe that if $f \in \mathcal{D}^{1,2}$, then $f \in L^{\frac{2 n}{n-2}, 2}$ with

$$
\|f\|_{\frac{2 n}{n-2}, 2} \leq C(n)\|\nabla f\|_{2} .
$$


This last inequality can be easily shown by a density argument. In fact, if $f \in$ $C_{0}^{\infty}\left(\mathbf{R}^{n}\right)$, then

$$
f(x)=\Gamma[\nabla f](x):=\frac{1}{n \omega_{n}} \int_{\mathbf{R}^{n}} \frac{x-y}{|x-y|^{n}} \cdot \nabla f(y) d y,
$$

where $\omega_{n}$ is the volume of the unit ball in $\mathbf{R}^{n}$. By using the Hardy-LittlewoodSobolev inequality it follows that the operator $\Gamma$ belongs to $\mathcal{L}\left(L^{p}, L^{q}\right)$ (the space of bounded linear operators from $L^{p}$ into $L^{q}$ ) for $1<p<n, 1<q<\infty$ and $1 / q=$ $1 / p-1 / n$. Then by real interpolation (the General Marcinkiewicz Interpolation Theorem 5.3.2 in Bergh and Löfström [5]) we obtain

$$
\Gamma \in \mathcal{L}\left(L^{2,2}, L^{\frac{2 n}{n-2}, 2}\right) .
$$

Finally, by using (10) and by recalling that $L^{2,2}=L^{2}$ we complete the proof of Lemma 2.1.

\section{Proof of Theorem 1.1}

We can now prove the main result of this paper. The proof is based on classical manipulations (see for instance J.-L. Lions [14], Ch. I) together with a new result, namely Eq. (11) below, that is obtained in Bae and Choe [1]. By a continuation argument we shall show that to prove Theorem 1.1 it is sufficient to prove the following lemma.

Lemma 3.1. Given a divergence-free $a_{0} \in H^{1}$, consider the Cauchy problem for the Navier-Stokes equations in $(\tau-\delta, \tau) \subseteq(0, T)$, with $a_{0}$ as initial value. Let $u$ be a weak solution in $(\tau-\delta, \tau)$, as well as a strong solution in $\left(\tau-\delta, \tau^{\prime}\right)$ for each $\tau^{\prime}<\tau$. Assume moreover that $\widehat{u}$ satisfies (9) for a small enough positive constant $C_{0}$. Under the above hypotheses $u$ is a strong (hence regular) solution in $(\tau-\delta, \tau)$.

Proof. We differentiate the first equation in (1) with respect to $x_{k}$, we take the scalar product with $\partial u_{i} / \partial x_{k}$ and we integrate over $\mathbf{R}^{n}$. We get

$$
\frac{1}{2} \frac{d}{d t} \int_{\mathbf{R}^{n}}|\nabla u|^{2} d x+\nu \int_{\mathbf{R}^{n}}\left|\nabla^{2} u\right|^{2} d x=-\int_{\mathbf{R}^{n}} \nabla[(u \cdot \nabla) u] \cdot \nabla u d x
$$

where

$$
|\nabla u|^{2}=\sum_{i, j=1}^{n}\left|\frac{\partial u_{i}}{\partial x_{j}}\right|^{2} \quad \text { and } \quad\left|\nabla^{2} u\right|^{2}=\sum_{i, j, k=1}^{n}\left|\frac{\partial^{2} u_{i}}{\partial x_{j} \partial x_{k}}\right|^{2} .
$$

By a careful inspection of the right hand side (see Eq. (2.3)-(2.4)-(2.5) in Beirão da Veiga [4]) it follows that

$$
\left|\int_{\mathbf{R}^{n}} \nabla[(u \cdot \nabla) u] \cdot \nabla u d x\right| \leq C_{2}(n) \int_{\mathbf{R}^{n}}|\widehat{u}||\nabla u|\left|\nabla^{2} u\right| d x,
$$


where $C_{2}(n)$ is a positive constant, depending only on the space dimension $n$. To prove (11) note that, by the incompressibility condition, we have

$$
\int_{\mathbf{R}^{n}} \nabla[(u \cdot \nabla) u] \cdot \nabla u d x=\int_{\mathbf{R}^{3}} \sum_{i, j, k=1}^{n} \frac{\partial u_{i}}{\partial x_{k}} \frac{\partial u_{j}}{\partial x_{i}} \frac{\partial u_{j}}{\partial x_{k}} d x
$$

If $i=j=n$, and by recalling that

$$
\frac{\partial u_{n}}{\partial x_{n}}=-\sum_{l=1}^{n-1} \frac{\partial u_{l}}{\partial x_{l}}
$$

we obtain that the right hand side of (12) is equal to

$$
-\int_{\mathbf{R}^{3}} \sum_{l=1}^{n-1} \sum_{k=1}^{n} \frac{\partial u_{n}}{\partial x_{k}} \frac{\partial u_{l}}{\partial x_{l}} \frac{\partial u_{n}}{\partial x_{k}} d x=\int_{\mathbf{R}^{3}} \sum_{l=1}^{n-1} u_{l} \sum_{k=1}^{n} \frac{\partial}{\partial x_{l}}\left[\frac{\partial u_{n}}{\partial x_{k}} \frac{\partial u_{n}}{\partial x_{k}}\right] d x .
$$

We then consider the case $i \neq n$

$$
\int_{\mathbf{R}^{3}} \sum_{i=1}^{n-1} \sum_{j, k=1}^{n} \frac{\partial u_{i}}{\partial x_{k}} \frac{\partial u_{j}}{\partial x_{i}} \frac{\partial u_{j}}{\partial x_{k}} d x=-\int_{\mathbf{R}^{n}} \sum_{i=1}^{n-1} u_{i} \sum_{j, k=1}^{n} \frac{\partial}{\partial x_{k}}\left[\frac{\partial u_{j}}{\partial x_{k}} \frac{\partial u_{j}}{\partial x_{i}}\right] d x
$$

Furthermore, if $i=n$ and $j \neq n$

$$
\int_{\mathbf{R}^{n}} \sum_{j=1}^{n-1} \sum_{k=1}^{n} \frac{\partial u_{n}}{\partial x_{k}} \frac{\partial u_{j}}{\partial x_{n}} \frac{\partial u_{j}}{\partial x_{k}} d x=-\int_{\mathbf{R}^{n}} \sum_{j=1}^{n-1} u_{j} \sum_{k=1}^{n} \frac{\partial}{\partial x_{k}}\left[\frac{\partial u_{n}}{\partial x_{k}} \frac{\partial u_{j}}{\partial x_{n}}\right] d x
$$

and (11) easily follows. By using (11) together with Lemma 2.1, we get the following estimate.

$$
\begin{aligned}
& \frac{1}{2} \frac{d}{d t} \int_{\mathbf{R}^{n}}|\nabla u|^{2} d x+\nu \int_{\mathbf{R}^{n}}\left|\nabla^{2} u\right|^{2} d x \\
& \quad \leq C_{1}(n) C_{2}(n) \sup _{\tau-\delta<t<\tau}[\widehat{u}(t)]_{n} \int_{\mathbf{R}^{n}}\left|\nabla^{2} u\right|^{2} d x .
\end{aligned}
$$

Finally, if

$$
\sup _{\tau-\delta<t<\tau}[\widehat{u}(t)]_{n} \leq \sup _{0<t<T}[\widehat{u}(t)]_{n}<\frac{\nu}{C_{1}(n) C_{2}(n)}:=C_{0}
$$

a standard application of the Gronwall lemma implies that

$$
\frac{1}{2} \int_{\mathbf{R}^{n}}|\nabla u|^{2}(t) d x \leq \frac{1}{2} \int_{\mathbf{R}^{n}}\left|\nabla a_{0}\right|^{2} d x, \quad t \in[\tau-\delta, \tau]
$$


This proves Lemma 3.1 if $n=3$, because $L^{\infty}\left(\tau-\delta, \tau ; H^{1}\right)$ is a regularity class. Observe that the Gronwall lemma implies also that

$$
\nu \int_{\tau-\delta}^{\tau} \int_{\mathbf{R}^{n}}\left|\nabla^{2} u\right|^{2} d x d t \leq \frac{1}{2} \int_{\mathbf{R}^{n}}\left|\nabla a_{0}\right|^{2} d x
$$

and, if $n=4$,

$$
u \in L^{2}\left(\tau-\delta, \tau ; H^{2}\left(\mathbf{R}^{4}\right)\right) \subset L^{2}\left(\tau-\delta, \tau ; W^{1,4}\left(\mathbf{R}^{4}\right)\right) .
$$

Since $W^{1,4}\left(\mathbf{R}^{4}\right) \not \subset L^{\infty}\left(\mathbf{R}^{4}\right)$, to conclude it is necessary to resort to the criterion proved in Beirão da Veiga [2]. In fact, in the last reference it is proved that if

$$
u \in L^{p}\left(\tau-\delta, \tau ; W^{1, q}\left(\mathbf{R}^{n}\right)\right) \text { for } \frac{2}{p}+\frac{n}{q} \leq 2 \text { with }<p \leq 2,
$$

then the weak solution $u$ is regular in $(\tau-\delta, \tau)$.

REMARK 3.2. If the external force $f$ does not vanishes, but if it is smooth enough (say for instance $f \in L^{2}\left(0, T ; L^{2}\right)$ and $\nabla \cdot f=0$ ), then Lemma 3.1 is still true. In fact, in this case the same manipulations as above imply that, for all $\eta>0$,

$$
\frac{1}{2} \frac{d}{d t} \int_{\mathbf{R}^{n}}|\nabla u|^{2} d x+\left[\nu-\frac{\eta}{2}-C_{1}(n) C_{2}(n) \sup _{\tau-\delta<t<\tau}[\widehat{u}(t)]_{n}\right] \leq \frac{1}{2 \eta} \int_{\mathbf{R}^{n}}|f|^{2} d x .
$$

By using Lemma 3.1 we can now easily prove Theorem 1.1.

Proof of Theorem 1.1. The proof of Theorem 1.1 follows by a continuation argument. In fact, the local existence theorem for strong solutions (see Leray [13] Chap. 4, §23, for the three-dimensional case and Kato [9] for the Cauchy problem in $\mathbf{R}^{4}$ ) implies that the Cauchy problem (1) has a unique strong solution in some interval $\left[0, T_{1}\right)$, for some strictly positive $T_{1}$. For convenience, let us suppose that this interval is the maximal interval of existence of the strong solution starting from $u_{0}$ at time $t=0$. Let us suppose that $T_{1}<T$. Then Lemma 3.1 with $a_{0}=u_{0}$ and $(\tau-\delta, \tau)=\left(0, T_{1}\right)$ shows that $u\left(T_{1}\right)$ belongs to $H^{1}$. A further continuation of $u$ is then possible. Absurd since $\left[0, T_{1}\right)$ was the maximal existence interval.

REMARK 3.3. We recall again that a strong solution is unique also in the larger class of Leray-Hopf weak solutions. In particular, to identify a weak solution on $[0, T]$ and a strong solution on the same interval, just the classical energy inequality (7) is necessary. We also observe that a more restrictive form of this inequality (roughly speaking the same for almost all $s \in[0, T]$ as initial values) is used in the proof of uniqueness of weak solutions with arbitrary norm in $L^{\infty}\left(0, T ; L^{n}\right)$.

REMARK 3.4. Observe that if $u_{0}$ belongs just to $L^{2}$ and if condition (9) is satisfied, then the solution is strong in $(t, T)$, for each $t>0$. 


\section{Some remarks on the problem in a general domain}

We observe that to obtain (11) it is essential to study the Navier-Stokes equations in the whole space, or at least in the periodic setting, to avoid the presence of boundary conditions. On the other hand, we may consider the Navier-Stokes equations in $\Omega$, where $\Omega \subset \mathbf{R}^{3}$ is a smooth (say $C^{2+\mu}$, for $\mu>0$ ) bounded domain.

We can prove in a different way the regularity-criterion involving the smallness of $u$ in the class (4), proved in Kozono [10]. We shall prove the following theorem, by assuming a condition that involves all the components of the velocity field. Recall that, since the initial-boundary value problem for the Navier-Stokes equations is generally supplemented with the homogeneous Dirichlet conditions

$$
u=0 \text { on } \partial \Omega,
$$

a weak solution must satisfy the following condition

$$
u \in C_{w}\left(0, T ; L^{2}(\Omega)\right) \cap L^{2}\left(0, T ; H_{0}^{1}(\Omega)\right),
$$

instead of (5). Furthermore, in (6) the test-functions must vanish on the boundary. For the existence of weak solution of the Navier-Stokes equations in such a domain $\Omega$, see for instance J.-L. Lions [14] or Masuda [16]. We have the following result.

Theorem 4.1 (Kozono). Let $u_{0} \in H_{0}^{1}(\Omega)$, with $\nabla \cdot u_{0}=0$ and let $u$ be $a$ weak solution of the Navier-Stokes equations in $\Omega \times(0, T)$, for $\Omega \subset \mathbf{R}^{3}$ as above. There exists a positive constant $C_{1}$ such that if

$$
\sup _{0<t<T}[u(t)]_{3}<C_{1}
$$

then the solution $u$ is strong in $(0, T)$.

Remark 4.2. By using the same techniques of Galdi and Maremonti [8] it is possible to prove the same result as Theorem 4.1 in a more general domain. In particular, it is sufficient that $\Omega$ is an arbitrary domain, uniformly of class $C^{2}$. It means that $\Omega$ lies on one part of its boundary $\partial \Omega$ and, for each $x_{0} \in \partial \Omega$, there exists a ball centered at $x_{0}$ and of radius independent of $x_{0}$, such that $\partial \Omega \cap B$ admits a Cartesian representation $x_{n}=\gamma\left(x_{1}, \ldots, x_{n}\right)$, where $\gamma$ is a function of class $C^{2}$ with derivatives up to the second bounded independently of $x_{0}$.

Reasoning as in the proof of Theorem 1.1, it is clear that to prove Theorem 4.1 it is enough to prove the following lemma.

Lemma 4.3. Given a divergence-free $a_{0} \in H_{0}^{1}(\Omega)$, consider the initial valueproblem for the Navier-Stokes equations in $\Omega \times(\tau-\delta, \tau) \subset \Omega \times(0, T)$, with $a_{0}$ as the initial value. Let $u$ be a weak solution of $(1)$ in $(\tau-\delta, \tau)$, as well as a strong 
solution in $\left(\tau-\delta, \tau^{\prime}\right)$ for each $\tau^{\prime}<\tau$. Assume moreover that $u$ satisfies (14) for a small enough positive constant $C_{1}$. Under the above hypotheses $u$ is a strong solution in $(\tau-\delta, \tau)$.

Proof. We use the classical manipulations introduced in Prodi [18], namely multiplying the equation by $A u$ (where $A$ is the well-known Stokes operator) and doing suitable integration by parts. Observe that the same estimate can be obtained by using the technique of the previous section.

It follows that

$$
\frac{1}{2} \frac{d}{d t} \int_{\Omega}|\nabla u|^{2} d x+\nu \int_{\Omega}|A u|^{2} d x \leq \int_{\Omega}|u\|\nabla u\| A u| d x .
$$

To estimate the right hand side of (15), we observe that if we work in a domain $\Omega$ as above of $\mathbf{R}^{3}$ the result of Lemma 2.1 is still true. In this case it is necessary to require, as additional hypothesis, that $v \in H_{0}^{1}(\Omega), w \in L^{2}(\Omega)$ and $z \in L_{w}^{3}(\Omega)$ to obtain (the norms and quasi-norms are obviously in $L^{p, q}(\Omega)$ )

$$
\left|\int_{\Omega} v w z d x\right| \leq C\|\nabla v\|_{2}\|w\|_{2}[z]_{3},
$$

see again Kozono [10]. We finally obtain

$$
\frac{1}{2} \frac{d}{d t} \int_{\Omega}|\nabla u|^{2} d x+\nu \int_{\Omega}|A u|^{2} d x \leq C \sup _{\tau-\delta<t<\tau}[u(t)]_{n} \int_{\Omega}|A u|^{2} d x
$$

and the same argument of Lemma 3.1 holds, since the Gronwall lemma implies that

$$
u \in L^{\infty}\left(\tau-\delta, \tau ; H_{0}^{1}(\Omega)\right)
$$

provided that

$$
\sup _{\tau-\delta<t<\tau}[u(t)]_{n}<C_{1}=\nu / C .
$$

This concludes the proof of Lemma 4.3.

\section{References}

[1] H.-O. Bae and H.-J. Choe, A regularity criterion for the Navier-Stokes equations, J. Math. Anal. Appl., to appear.

[2] H. Beirão da Veiga, A new regularity class for the Navier-Stokes equations in $\mathbf{R}^{n}$, Chinese Ann. Math. Ser. B, 16 (4) (1995), 407-412.

[ 3 ] H. Beirão da Veiga, Remarks on the smoothness of the $L^{\infty}\left(0, T ; L^{3}\right)$ solutions of the 3 -I) Navier-Stokes equations, Portugal. Math., 54 (4) (1997), 381-391.

[4] H. Beirão da Veiga, On the smoothness of a class of weak solutions to the Navier-Stokes equations, J. Math. Fluid Mech., 2 (4) (2000), 315-323. 
[5] J. Bergh and J. Löfström, Interpolation spaces. An introduction, Springer-Verlag, Berlin, 1976, Grundlehren der Mathematischen Wissenschaften, No. 223.

[6] L. Caffarelli, R. Kohn and L. Nirenberg, Partial regularity of suitable weak solutions of the Navier-Stokes equations, Comm. Pure Appl. Math., 35 (6) (1982), 771-831.

[7] G.P. Galdi, An introduction to the Navier-Stokes initial-boundary value problem, Fundamental directions in mathematical fluid mechanics, Adv. Math. Fluid Mech., pp. 1-70, Birkhäuser, Basel, 2000.

[8] G.P. Galdi and P. Maremonti, Regularity of weak solutions of the Navier-Stokes system in arbitrary domains, Ann. Univ. Ferrara Sez. VII (N.S.), 34 (1988), 59-73.

[9] T. Kato, Strong $L^{p}$-solutions of the Navier-Stokes equation in $\mathbf{R}^{m}$, with applications to weak solutions, Math. Z., 187 (4) (1984), 471-480.

[10] H. Kozono, Uniqueness and regularity of weak solutions to the Navier-Stokes equations, Recent topics on mathematical theory of viscous incompressible fluid (Tsukuba, 1996), Lecture Notes Number Appl. Anal. 16, pp. 161-208, Kinokuniya, Tokyo, 1998.

[11] H. Kozono and H. Sohr, Remark on uniqueness of weak solutions to the Navier-Stokes equations, Analysis, 16 (3) (1996), 255-271.

[12] H. Kozono and H. Sohr, Regularity criterion on weak solutions to the Navier-Stokes equations, Adv. Differential Equations, 2 (4) (1997), 535-554.

[13] J. Leray, Sur le mouvement d'un liquide visqueux emplissant l'espace, Acta Math., 63 (1934), 193-248.

[14] J.-L. Lions, Quelques méthodes de résolution des problèmes aux limites non linéaires, Dunod Gauthier-Villars, Paris, 1969.

[15] P.-L. Lions and N. Masmoudi, Uniqueness of mild solutions of the Navier-Stokes system in $L^{N}$, Comm. Partial Differential Equations, 26 (2001), 2211-2226.

[16] K. Masuda, Weak solutions of Navier-Stokes equations, Tôhoku Math. J., 36 (4) (1984), 623-646.

[17] J. Nečas, M. Růžička and V. Šverák, On Leray's self-similar solutions of the Navier-Stokes equations, Acta Math., 176 (2) (1996), 283-294.

[18] G. Prodi, Un teorema di unicità per le equazioni di Navier-Stokes, Ann. Mat. Pura Appl., 48 (4) (1959), 173-182.

[19] J. Serrin, The initial value problem for the Navier-Stokes equations, Nonlinear Problems Proc. Sympos., Madison, Wis., pp. 69-98, Univ. of Wisconsin Press, Madison, Wis., 1963.

[20] H. Sohr, Zur Regularitätstheorie der instationären Gleichungen von Navier-Stokes, Math. Z., 184 (3) (1983), 359-375.

[21] H. Sohr and W. von Wahl, On the singular set and the uniqueness of weak solutions of the Navier-Stokes equations, Manuscripta Math., 49 (1) (1997), 27-59.

[22] M. Struwe, On partial regularity results for the Navier-Stokes equations, Comm. Pure Appl. Math., 41 (4) (1988), 437-458.

Dipartimento di Matematica

$$
\text { Applicata "U. Dini" }
$$

V. Le Bonanno 25/B, 56126 Pisa, Italy

E-mail: berselli@dma.unipi.it 